\title{
Macroscopic Periodic Tunneling of Fermi Atoms in the BCS-BEC Crossover
}

\author{
L. Salasnich ${ }^{1,3}$, N. Manini ${ }^{2}$ and F. Toigo ${ }^{3}$ \\ ${ }^{1}$ CNR-INFM, Via Marzolo 8, 35131 Padova, Italy \\ ${ }^{2}$ Dipartimento di Fisica and CNISM, Università di Milano, Via Celoria 16, 20133 Milano, Italy \\ ${ }^{3}$ Dipartimento di Fisica "Galileo Galilei" and CNISM, \\ Università di Padova, Via Marzolo 8, 35131 Padova, Italy
}

\begin{abstract}
We study the macroscopic quantum tunneling of two weakly-linked superfluids made of interacting fermionic atoms. We derive atomic Josephson junction equations and find that zero-mode and $\pi$ mode frequencies of coherent atomic oscillations depend on the tunneling coefficient and the sound velocity of the superfluid. By considering a superfluid of ${ }^{40} \mathrm{~K}$ atoms, we calculate these oscillation frequencies in the crossover from the Bardeen-Cooper-Schrieffer state of weakly-bound Cooper pairs to the Bose-Einstein Condensate of strongly-bound molecular dimers.
\end{abstract}

PACS numbers: 03.75.Lm, 03.75.Ss, 05.30.Jp, 74.50.+r

\section{INTRODUCTION}

The crossover from the Bardeen-Cooper-Schrieffer (BCS) state of Cooper fermion pairs to the Bose-Einstein condensate (BEC) of molecular dimers with ultra-cold two-hyperfine-components Fermi vapors of ${ }^{40} \mathrm{~K}$ atoms [1, 2, 3] and ${ }^{6} \mathrm{Li}$ atoms [4, 5, 6] has been observed in the last years by several experimental groups with the use of Fano-Feshbach resonances [7]. From the theoretical side, extended BCS (EBCS) equations 8, 9, 10] have been used to reproduce density profiles [11] and collective oscillations [12, 13] of these Fermi gases. In addition, more recent calculations based on Monte Carlo (MC) fitting and superfluid dynamics [14] have shown that the mean-field EBCS theory is quite accurate.

Recently, Spuntarelli et al. [15] studied the stationary Josephson effect [16] across the BCS-BEC crossover with neutral fermions by using the EBCS equations: in detail, they computed the current-phase relation throughout the BCS-BEC crossover at zero temperature for a two-spin component Fermi gas in the presence of a barrier. The Josephson effect of atomic superfluids, i.e. coherent oscillations between two weakly-linked bosonic clouds, was predicted [17] and observed [18] with BECs. Josephson oscillations in superfluid atomic Fermi gases have been thoretically considered by Paraoanu et al. [19], Wouters et al. [20], and Adhikari [21]. The macroscopic oscillations of tunneling neutral atoms is closely related to the familiar Josephson effect of charged electrons in superconductor junctions [22] and its investigation can help a deeper understanding of these exotic states of matter.

In this work we present an investigation of the dynamical Josephson effect in the BCS-BEC crossover based on a time dependent local density approximation for the dynamics of the Ginzburg-Landau (GL) order parameter of the atomic Cooper pairs [23], starting from a reliable parameterization of the bulk chemical potential 14] in the crossover. From our zero-temperature GL equation we obtain the atomic Josephson junction equations for two weekly linked fermionic superfluids. The main result provides zero-mode and $\pi$-mode Josephson frequencies of periodic quantum tunneling as a function of the sound velocity. Our investigation of the Josephson effect with neutral Fermi atoms, a direct manifestation of macroscopic quantum phase coherence, is of interest not only conceptually but also for future applications in quantum computing [24, 25].

\section{GINZBURG-LANDAU EQUATION AT ZERO TEMPERATURE}

In a dilute Fermi gas of $N$ atoms with two equally populated spin components and attractive inter-atomic strength at zero temperature, superfluidity and coherence are strongly related to the properties of the twoparticle density matrix. Let $\hat{\psi}_{\sigma}(\mathbf{r}, t)$ be the field operator that destroys a fermion of $\operatorname{spin} \sigma(\sigma=\uparrow, \downarrow)$ in the position $\mathbf{r}$ at time $t$. The two-particle density matrix can be written as

$$
\begin{aligned}
& \left\langle\hat{\psi}_{\sigma_{1}}^{+}\left(\mathbf{r}_{1}, t\right) \hat{\psi}_{\sigma_{2}}^{+}\left(\mathbf{r}_{2}, t\right) \hat{\psi}_{\sigma_{1}^{\prime}}\left(\mathbf{r}_{2}^{\prime}, t\right) \hat{\psi}_{\sigma_{2}^{\prime}}\left(\mathbf{r}_{2}^{\prime}, t\right)\right\rangle= \\
& \sum_{j} N_{j}(t) \chi_{j}^{*}\left(\mathbf{r}_{1} \sigma_{1}, \mathbf{r}_{2} \sigma_{2}, t\right) \chi_{j}\left(\mathbf{r}_{1}^{\prime} \sigma_{1}^{\prime}, \mathbf{r}_{2}^{\prime} \sigma_{2}^{\prime}, t\right)
\end{aligned}
$$

where $\langle\cdot\rangle$ is the ground-state average, $\chi_{j}$ and $N_{j}$ are respectively normalized eigenfunctions and eigenvalues of the two-particle density matrix, and the maximum eigenvalue cannot exceed $N / 2$ [9, 26]. If one eigenvalue, say $N_{0}$, is of order $N / 2$, off-diagonal long-range order is present.

At zero temperature, where the superfluid density coincides with the total density, the GL order parameter describing the motion of Cooper pairs of atoms is defined as

$$
\Psi(\mathbf{r}, t)=\sqrt{\frac{N}{2}} \chi_{0}(\mathbf{r} \uparrow, \mathbf{r} \downarrow, t)=\sqrt{\frac{n(\mathbf{r}, t)}{2}} \exp (i \theta(\mathbf{r}, t)),
$$

where $n(\mathbf{r}, t)$ is the local atomic number density $(n(\mathbf{r}, t) / 2$ is the local density of pairs) and $\theta(\mathbf{r}, t)$ is precisely the phase of the condensate wave function $\chi_{0}[9,23,27]$. 
Note that $\theta(\mathbf{r}, t)$ is also the phase of the gap function $\Delta(\mathbf{r}, t)=|\Delta(\mathbf{r}, t)| \exp (i \theta(\mathbf{r}, t))$ of Cooper pairs $[9,23,26,27]$. Under an external potential $U(\mathbf{r})$ acting on individual atoms, the low-energy collective properties of the Fermi superfluid [14] can be described by the the following highly nonlinear time-dependent GL equation (TDGLE)

$$
i \hbar \frac{\partial}{\partial t} \Psi(\mathbf{r}, t)=\left[-\frac{\hbar^{2}}{4 m} \nabla^{2}+2 U(\mathbf{r})+2 \mu(n(\mathbf{r}))\right] \Psi(\mathbf{r}, t) .
$$

Here $m$ is the mass of one atom and

$$
\mu(n)=\frac{\partial}{\partial n}(n \mathcal{E}(n))
$$

is the atomic bulk chemical potential of a homogeneous fluid with density $n$ [28], if $\mathcal{E}(n)$ is its energy per particle. The phase of the order parameter drives the superfluid velocity

$$
\mathbf{v}(\mathbf{r}, t)=\frac{\hbar}{2 m} \nabla \theta(\mathbf{r}, t),
$$

which is irrotational $(\nabla \wedge \mathbf{v}=0)$ by construction [28]. The equation of superfluid velocity permits to map Eq. (3) to the hydrodynamic equations of fermionic superfluids:

$$
\begin{gathered}
\frac{\partial n}{\partial t}+\nabla \cdot(n \mathbf{v})=0 \\
m \frac{\partial \mathbf{v}}{\partial t}+\nabla\left[-\frac{\hbar^{2}}{8 m} \frac{\nabla^{2} \sqrt{n}}{\sqrt{n}}+\frac{m}{2} v^{2}+U+\mu(n)\right]=0
\end{gathered}
$$

In these zero-temperature hydrodynamical equations, which are valid in the full BCS-BEC crossover to describe macroscopic long-wavelength phenomena of the fermionic superfluid 29], statistics enters through the equation of state $\mu(n)$ and the quantum-pressure term $-\left[\hbar^{2} /(8 m \sqrt{n})\right] \nabla^{2} \sqrt{n}$, which is absent in the classical hydrodynamic equations [28, 29]. The coefficient $1 / 8$ in the quantum pressure holds for pairs of fermions, as opposed to atomic bosons, where the coefficient is $1 / 2$, and $\mathbf{v}=(\hbar / m) \nabla \theta$. The bulk chemical potential $\mu(n)$ is the key ingredient of our study; theoretical calculations in both asymptotic limits of $1 / y$ and Montecarlo data 30] suggest that in the BCS-BEC crossover it can be written as

$$
\mu(n)=\frac{\hbar^{2}}{2 m}\left(3 \pi^{2} n\right)^{2 / 3}\left[\epsilon(y)-\frac{y}{5} \epsilon^{\prime}(y)\right],
$$

where $\epsilon(y)$ is a dimensionless universal function of the inverse interaction parameter $y=1 / k_{F} a_{F}$, where $k_{F}=$ $\left(3 \pi^{2} n\right)^{-1 / 3}$ is the Fermi wavenumber of the noninteracting fermions and $a_{F}$ is the fermion-fermion scattering length. The function $\epsilon(y)$ was parameterized across the BCS-BEC crossover by Manini and Salasnich [14] to fit the MC data of Astrakharchik et al. [30] and the asymptotic expressions of the bulk energy per particle

$$
\mathcal{E}=\frac{3}{5} \epsilon_{F} \epsilon(y),
$$

with $\epsilon_{F}=\left(\hbar^{2} k_{F}^{2} / 2 m\right)$ the Fermi energy and $k_{F}=$ $\left(3 \pi^{2} n\right)^{1 / 3}$ the Fermi wave number. The parametrization of $\epsilon(y)$ chosen in Ref. [14] is the following

$$
\epsilon(y)=\alpha_{1}-\alpha_{2} \arctan \left(\alpha_{3} y \frac{\beta_{1}+|y|}{\beta_{2}+|y|}\right),
$$

where the values of the parameters $\alpha_{1}, \alpha_{2}, \alpha_{3}, \beta_{1}, \beta_{2}$ are reported in that work. In the BEC regime $(y \gg 1)$, where $\mu(n) \sim n$, the TDGLE reduces to the time-dependent Gross-Pitaevskii equation for composite bosons of mass $2 m$ subject to the effective potential $2 U(\mathbf{r})+2 \mu(n(\mathbf{r}, t))$ [15]. In the BCS regime $(y \ll-1)$, where $\mu(n) \sim$ $\epsilon_{F}\left(1+k_{F} a_{F} /(3 \pi)\right)$, the TDGLE gives the AndersonBogoliubov mode (sound velocity) of neutral superconductors.

Some years ago an effective nonlinear Schrödinger equation (NLSE) for superconductors was derived from the microscopic BCS Lagrangian in the low-frequency long-wavelength limit of $|\Delta(\mathbf{r}, t)|$ by Aitchison et al. and De Palo et al. 31]. That NLSE and our TDGLE produce the same Anderson-Bogoliubov mode [14, 31], but TDGLE describes accurately sound velocity and other collective modes of the fermionic superfluid in the full BCS-BEC crossover [14].

\section{DYNAMICS OF TWO WEAKLY-LINKED FERMI SUPERFLUIDS}

Assume that $U(\mathbf{r})$ is a potential with a barrier that splits the superfluid into two subsystems A and B separated by a region $\mathrm{C}$ where the modulus of the order parameter is exponentially small [32]. We look for a timedependent solution of the TDGLE of the form

$$
\Psi(\mathbf{r}, t)=\Psi_{\mathrm{A}}(t) \Phi_{\mathrm{A}}(\mathbf{r})+\Psi_{\mathrm{B}}(t) \Phi_{\mathrm{B}}(\mathbf{r}),
$$

where $\Phi_{\alpha}(\mathbf{r})$ is the quasi-stationary solution (real and normalized to unity) of the TDGLE localized in region $\alpha$ $(\alpha=\mathrm{A}, \mathrm{B})$.

By inserting this ansatz for $\Psi$ into Eq. (3), after integration over space and neglecting exponentially small $\Phi_{A} \Phi_{B}$ terms, the system can be described by the following two-state model [33]:

$$
\begin{aligned}
i \hbar \frac{\partial}{\partial t} \Psi_{\mathrm{A}} & =E_{\mathrm{A}} \Psi_{\mathrm{A}}-K \Psi_{\mathrm{B}} \\
i \hbar \frac{\partial}{\partial t} \Psi_{\mathrm{B}} & =E_{\mathrm{B}} \Psi_{\mathrm{B}}-K \Psi_{\mathrm{A}}
\end{aligned}
$$

for the two complex coefficients $\Psi_{\alpha}(t)$. Here $E_{\alpha}=E_{\alpha}^{0}+$ $E_{\alpha}^{I}$ is the energy in region $\alpha$, with

$$
E_{\alpha}^{0}=\int \Phi_{\alpha}\left[-\frac{\hbar^{2}}{4 m} \nabla^{2}+2 U\right] \Phi_{\alpha} d^{3} \mathbf{r}
$$

and

$$
E_{\alpha}^{I} \simeq \int \Phi_{\alpha} 2 \mu\left(2\left|\Psi_{\alpha}\right|^{2} \Phi_{\alpha}^{2}\right) \Phi_{\alpha} d^{3} \mathbf{r}
$$


The coupling energy $K$ describes phenomenologically the tunneling between the two regions. It is quite common to use a phenomenological tunneling energy to analyze the Josephson effect in superconductors 9, 22, 27, 33]. In general, thermal [34] and quantum [35] fluctuations will affect the value of $K$.

At zero-temperature the single-particle tunneling energy $t_{0}$ can be estimated as

$$
t_{0} \simeq-\frac{1}{2} \int \Phi_{\mathrm{A}}\left[-\frac{\hbar^{2}}{4 m} \nabla^{2}+2 U\right] \Phi_{\mathrm{B}} d^{3} \mathbf{r},
$$

if the barrier is orthogonal to the $x$ axis and such that $\int_{C}\left[\frac{8 m}{\hbar^{2}}(U(x)-\mu(n))\right]^{1 / 2} d x \gg 1$. In the $\mathrm{BEC}$ region the quantum depletion is negligible [26], the tunneling is fully coherent, and the coupling energy is simply $K \simeq 2 t_{0}$ [17, 35]. Instead in the deep BCS regime, where there is a very large quantum depletion, only a small fraction of pairs perform coherent tunneling, and microscopic calculations suggest $K \simeq|\Delta| t_{0}^{2} /\left(8 \pi \epsilon_{F}^{2} N\right)$ [36]. Unfortunately a microscopic derivation of $K$ in the full BCS-BEC crossover is not yet available.

Under the assumption that the double-well potential $U(\mathbf{r})$ has the shape of two weakly-linked sharp-edged boxes of volumes $V_{\mathrm{A}}$ and $V_{\mathrm{B}}$, we can write $\Psi_{\alpha}(t)=$ $\sqrt{N_{\alpha}(t) / 2} \exp \left(i \theta_{\alpha}(t)\right)$, where $N_{\alpha}(t)$ and $\theta_{\alpha}(t)$ are the number of fermions and the phase in region $\alpha$. In terms of the phase difference

$$
\varphi(t)=\theta_{\mathrm{B}}(t)-\theta_{\mathrm{A}}(t)
$$

and relative number imbalance

$$
z(t)=\frac{N_{\mathrm{A}}(t)-N_{\mathrm{B}}(t)}{N},
$$

with $N=N_{\mathrm{A}}(t)+N_{\mathrm{B}}(t)$ the constant total number of atoms, Eqs. (12) and (13) give

$$
\begin{aligned}
\dot{z} & =-\frac{2 K}{\hbar} \sqrt{1-z^{2}} \sin \varphi, \\
\dot{\varphi} & =\frac{2}{\hbar}\left[\mu\left(\frac{N}{2 V_{\mathrm{A}}}(1+z)\right)-\mu\left(\frac{N}{2 V_{\mathrm{B}}}(1-z)\right)\right] \\
& +\frac{2 K}{\hbar} \frac{z}{\sqrt{1-z^{2}}} \cos \varphi+\frac{E_{\mathrm{A}}^{0}-E_{\mathrm{B}}^{0}}{\hbar} .
\end{aligned}
$$

These are the atomic Josephson junction (AJJ) equations for the two dynamical variables $z(t)$ and $\varphi(t)$ describing the oscillations of $N$ Fermi atoms tunneling in the superfluid state between region $A$ of volume $V_{\mathrm{A}}$ and region $B$ of volume $V_{\mathrm{B}}$. These equations, linking the tunneling current $I=-\dot{z} N / 2=(K N / \hbar) \sqrt{1-z^{2}} \sin \varphi=$ $I_{0} \sqrt{1-z^{2}} \sin \varphi$ to the phase difference $\varphi$, reduce to the familiar Josephson's expression $I=I_{0} \sin (\varphi)$, well established for BCS superconductors, in the appropriate limit $|z| \ll 1$ [22]. Also, in the deep BEC regime, where $\mu(n) \sim$ $n$, the AJJ equations reduce to the bosonic Josephson junction (BJJ) equations introduced by Smerzi et al. [17] and found to describe accurately the density oscillations of weakly linked condensates [18].

\section{AJJ EQUATIONS: LINEAR REGIME}

The AJJ equations can be solved across the BCS-BEC crossover, even taking into account a small but finite imbalance $z$. To first order in $z$, Eqs. (19) and (20) read

$$
\begin{aligned}
& \dot{z}=-\frac{2 K}{\hbar} \sin \varphi \\
& \dot{\varphi}=\frac{2 \Lambda}{\hbar} z+\frac{2 K}{\hbar} z \cos \varphi+\frac{2 \tilde{\mu}_{\mathrm{AB}}}{\hbar},
\end{aligned}
$$

where

$$
\Lambda=\frac{N}{2}\left[\left.\frac{1}{V_{\mathrm{A}}} \frac{\partial \mu}{\partial n}\right|_{\mathrm{A}}+\left.\frac{1}{V_{\mathrm{B}}} \frac{\partial \mu}{\partial n}\right|_{\mathrm{B}}\right],
$$

and

$$
\tilde{\mu}_{\mathrm{AB}}=\frac{1}{2}\left(\left.\mu\right|_{\mathrm{A}}-\left.\mu\right|_{\mathrm{B}}\right)+\left(E_{\mathrm{A}}^{0}-E_{\mathrm{B}}^{0}\right),
$$

with $\left.\mu\right|_{\alpha}$ and $\left.\frac{\partial \mu}{\partial n}\right|_{\alpha}$ the bulk chemical potential and its derivative are calculated at the density $N /\left(2 V_{\alpha}\right), \alpha=$ $A, B$.

In the symmetric case $\left(V_{\mathrm{A}}=V_{\mathrm{B}}=V / 2\right.$ and $\left.E_{A}^{0}=E_{B}^{0}\right)$ $\Lambda$ takes the particularly simple form

$$
\Lambda=2 n \frac{\partial \mu}{\partial n}=2 m c_{s}^{2}
$$

where $c_{s}$ is the sound velocity computed at the mean density $n=N / V$ of the superfluid. ¿From Eq. (8) one has thus

$$
\Lambda=2 m v_{F}^{2}\left[\frac{1}{3} \epsilon(y)-\frac{y}{5} \epsilon^{\prime}(y)+\frac{y^{2}}{30} \epsilon^{\prime \prime}(y)\right],
$$

where $v_{F}=\hbar k_{F} / m$ is the Fermi velocity of noninteracting fermions.

In the symmetric case $\tilde{\mu}_{A B}=0$, Eqs. (21) and (22) admit a stable stationary solution $(\bar{z}, \bar{\varphi})$ with $\bar{z}=0$ and $\bar{\varphi}=2 \pi j$, for integer $j$. If $\Lambda / K<1$, also $\bar{z}=0$ and $\bar{\varphi}=\pi(2 j+1)$ is a stable stationary solution. These stationary solutions remain stable even if one includes higher-order terms in the $z$-expansion of Eqs. (19) and (20). Small oscillations around the stable solutions with $\bar{\varphi}=2 \pi j$ and $\bar{\varphi}=\pi(2 j+1)$ have frequencies

$$
\nu_{0 / \pi}=\frac{K}{\pi \hbar} \sqrt{1 \pm \frac{\Lambda}{K}}
$$

and are called zero-mode (with + ) and $\pi$-mode (with -), respectively. The zero-mode is the analog of the Josephson plasma frequency in superconducting junctions [22]. The analogous of this $\pi$-mode was observed in weakly coupled reservoirs of ${ }^{3} \mathrm{He}-\mathrm{B}[37]$ and discussed in the BJJ equations [17.

By using the functional dependence on the inverse interaction parameter $y=1 /\left(k_{F} a_{F}\right)$ of the sound velocity $c_{s}$, and inserting this expression of $\Lambda$ in Eq. (27), we obtain the oscillation frequencies $\nu_{0}$ and $\nu_{\pi}$. As an example, 


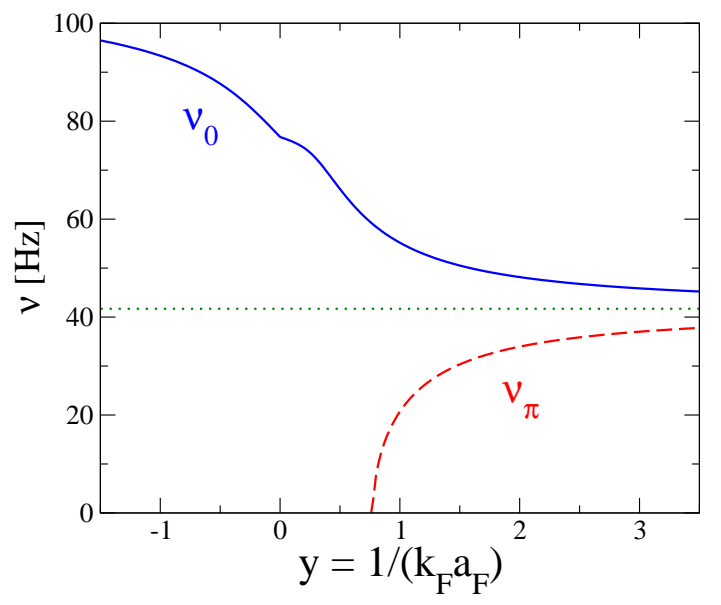

FIG. 1: (color online). Zero-mode frequency $\nu_{0}$ (solid) and $\pi$ mode frequency $\nu_{\pi}$ (dashed) for a superfluid of $N=10^{6}{ }^{40} \mathrm{~K}$ atoms between two symmetric regions of volume $25 \cdot 10^{6} \mu \mathrm{m}^{3}$ (mean density $n=0.02 \mu \mathrm{m}^{-3}$ ) and fixed tunneling parameter $K / k_{\mathrm{B}}=10^{-9}$ Kelvin.

consider a fermionic superfluid of ${ }^{40} \mathrm{~K}$ atoms with total density $n=0.02$ atoms $/ \mu \mathrm{m}^{3}$ : Fig. 1 reports $\nu_{0 / \pi}$ as a function of $y$, assuming that the tunneling energy can be held fixed to the value $K / k_{\mathrm{B}}=10^{-9}$ Kelvin (where $k_{\mathrm{B}}$ is the Boltzmann constant). As previously stressed in the deep BCS regime $K$ is proportional to $|\Delta|$ and becomes rapidly very small, thus in practice it would be difficult to keep constant. The zero-mode frequency decreases for increasing $y$ across the crossover, approaching the asymptotic value $K /(\pi \hbar)$ (dotted line) in the BEC limit $(y \gg 1)$. In contrast, the $\pi$-mode does not exist until past the unitarity point $y=0$, i.e. until the value of $\Lambda$ becomes smaller than $K$; then $\nu_{\pi}$ increases approaching the same large- $y$ limit as $\nu_{0}$.

In limiting cases of the BCS-BEC crossover analytical expressions are available for $\Lambda$. (i) Deep BCS regime $(y \ll-1)$ : the sound velocity $c_{s}$ approaches $v_{F} / \sqrt{3}$ [14], and $\Lambda=2 m v_{F}^{2}=2\left(\hbar^{2} / m\right)\left(3 \pi^{2} n\right)^{2 / 3}$. (ii) Unitarity point $(y=0)$ : according to $\mathrm{MC}$ simulations of Astrakharchik et al. [30] the sound velocity is $c_{s} \simeq 0.37 v_{F}$, thus $\Lambda=0.29 m v_{F}^{2}$. (iii) Deep BEC regime: all molecular dimers are in the BEC and the sound velocity is such that $m c_{s}^{2}=\pi \hbar^{2} a_{M} n / m$ [14], where $a_{M}=0.6 a_{F}$ is the dimer-dimer scattering length [30]. In this limit $a_{F} \rightarrow 0^{+}$for non-interacting bosonic dimers, $\Lambda=0$ and corresponding $\nu_{0}=\nu_{\pi}=K /(\pi \hbar)$.

\section{AJJ EQUATIONS: NONLINEAR EFFECTS}

We come now to nonlinear effects in the AJJ equations (19) and (20) in the symmetric case $\left(V_{A}=V_{B}=V / 2\right.$ and $\left.E_{A}^{0}=E_{B}^{0}\right)$. The variables $\varphi$ and $z$ are canonically

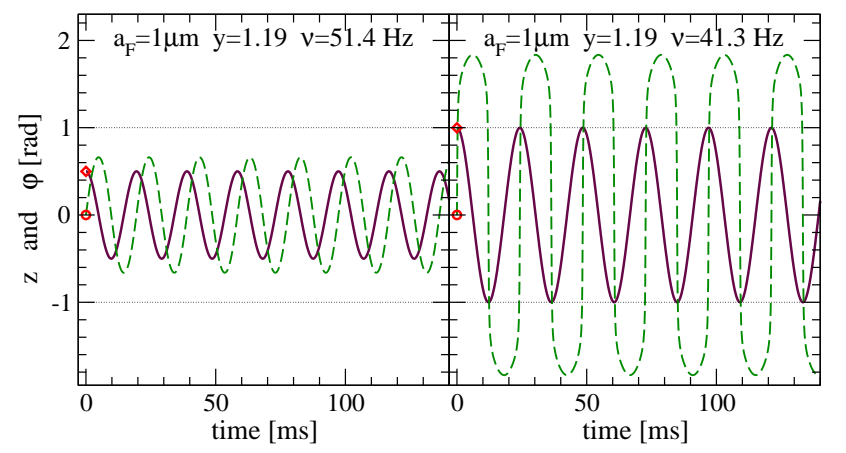

FIG. 2: (color online). Nonlinear effects on the zero-mode oscillation of $N=10^{6}{ }^{40} \mathrm{~K}$ atoms between two symmetric regions with the same conditions of Fig. 1 at fixed interaction $a_{F}=1 \mu \mathrm{m}$, corresponding to $y=1.19$. These trajectories result from the integration of the coupled equations (19) and (20) for $z(t)$ (solid) and $\varphi(t)$ (dashed), with initial conditions $\varphi(0)=0$, and $z(0)=0.5$ (left) or $z(0)=0.999$ (right).

conjugate, with

$$
\begin{aligned}
& \dot{z}=-\frac{\partial H}{\partial \varphi}, \\
& \dot{\varphi}=\frac{\partial H}{\partial z},
\end{aligned}
$$

where the Hamiltonian is

$$
H=\frac{2}{\hbar} G(z)-\frac{2 K}{\hbar} \sqrt{1-z^{2}} \cos \varphi,
$$

with

$$
G^{\prime}(z)=F(z)=\mu\left(\frac{N}{V}(1+z)\right)-\mu\left(\frac{N}{V}(1-z)\right) .
$$

It is straightforward to show that these nonlinear equations admit a symmetry-breaking stationary solution $(\bar{z}, \bar{\varphi})$, with $\bar{\varphi}=\pi(2 j+1)$ and with $\bar{z}$ the solution of the equation $F(z)=K z / \sqrt{1-z^{2}}$. The analysis of stability shows that this symmetry-breaking solution, where the superfluid displays macroscopic self-trapping, is stable only if $K>F^{\prime}(\bar{z})\left(1-\bar{z}^{2}\right)^{3 / 2}$.

To analyze nonlinear effects we solve numerically the AJJ equations. Regular zero-mode oscillations of $z(t)$ and $\varphi(t)$ are displayed in Fig. 2, under the same conditions as for Fig. [1. The oscillation starting from $z(0)=$ 0.5 indicates that the solution (27) of the linearized equations (21) and (22) are fairly accurate even for finite and not quite small amplitude. Eventually however, for very large amplitude, $z(0)=0.999$, deviations from the harmonic approximation become quite visible. These deviations are illustrated, for increasing amplitude, in Fig. 3. the left panel shows that the frequencies of the zero- and $\pi$-modes approach each other. The right panel shows the decay in the fractional leading Fourier component of the oscillation $\varphi(t)$, as the wave shape distorts from a perfect sinusoid, and acquires higher (odd) Fourier components. Note the extremely small deformations from 


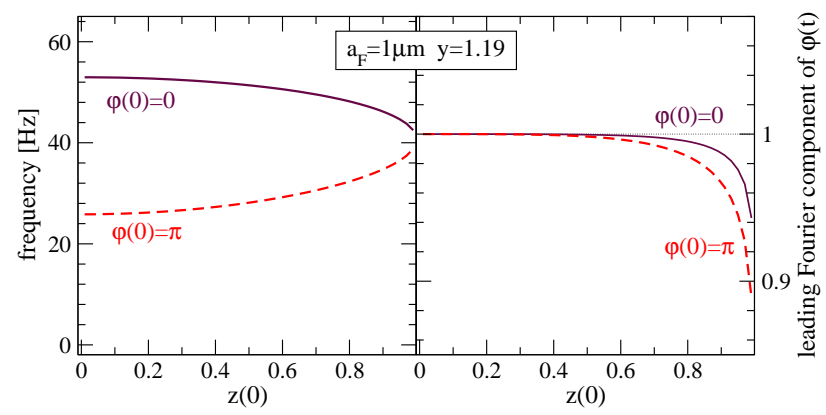

FIG. 3: (color online). Nonlinear effects on the zero-mode (solid) and $\pi$-mode (dashed) frequency (left) and waveform (right) for the same conditions as in Fig. 2, as functions of the initial amplitude $z(0)$. In an expansion $\varphi(t)=\varphi(0)+$ $\sum_{k=1}^{\infty} a_{k} \sin (2 \pi \nu k t)$, the fractional leading Fourier component of the right panel is $a_{1}^{2} / \sum_{k} a_{k}^{2}$.

perfect harmonic oscillations and the minor deviations of the frequencies from the linear values $\nu_{0}=53.0 \mathrm{~Hz}$ and $\nu_{\pi}=25.8 \mathrm{~Hz}$ of Eq. (27), until $z(0) \simeq 0.6$. Similar evolutions near the $y=0$ point on the BEC side, and on the BCS side, show oscillations of $z(t)$ around the self-trapping symmetry-breaking stationary solution $\bar{z}$, accompanied by monotonously increasing phase $\varphi(t)$.

\section{CONCLUSIONS}

We have investigated the macroscopic quantum tunneling of two weakly-linked Fermi superfluids in the BCS-
BEC crossover by using AJJ equations. We remark that the coupling energy $K$ appearing in our Josephson equations is a phenomenological parameter: from the experimental measurement of the frequencies of periodic quantum tuneling one can infer the value of $K$ by using Eq. (27). Analytical expression of $K$ based on microscopic theory are available only in the deep BCS regime and in the deep BEC regime. An important issue is surely the development of a microscopic theory of tunneling in the full BEC-BEC crossover. We also stress that all Josephson oscillatory frequencies discussed here cannot exceed the frequency $|\Delta| /(\pi \hbar)$, associated to the breaking of Cooper pairs. The AJJ equations can be extended to investigate Josephson junction arrays for neutral fermionic atoms in optical lattices, potentially of extreme relevance for quantum information and quantum computing with ultracold atoms.

\section{Acknowledgments}

This work has been partially supported by Fondazione CARIPARO and by the EU's 6th Framework Programme through contract NMP4-CT-2004-500198. L.S. has been partially supported by GNFM-INdAM and thanks A. Bulgac, B.A. Malomed, A. Minguzzi, A. Parola, P. Pieri, A. Smerzi and A. Trombettoni for useful discussions.
[1] M. Greiner, C.A. Regal, and D.S. Jin, Nature (London) 426, 537 (2003).

[2] C.A. Regal, M. Greiner, and D.S. Jin, Phys. Rev. Lett. 92, 040403 (2004).

[3] J. Kinast, S.L. Hemmer, M.E. Gehm, A. Turlapov, and J.E. Thomas, Phys. Rev. Lett. 92, 150402 (2004).

[4] M.W. Zwierlein, C.A. Stan, C.H. Schunck, S.M.F. Raupach, A.J. Kerman, and W. Ketterle, Phys. Rev. Lett. 92, 120403 (2004); M.W. Zwierlein, C.H. Schunck, C.A. Stan, S.M.F. Raupach, and W. Ketterle, Phys. Rev. Lett. 94, 180401 (2005).

[5] C. Chin, M. Bartenstein, A. Altmeyer, S. Riedl, S. Jochim, J.H. Denschlag, and R. Grimm, Science 305, 1128 (2004); M. Bartenstein, A. Altmeyer, S. Riedl, S. Jochim, C. Chin, J.H. Denschlag, and R. Grimm, Phys. Rev. Lett. 92, 203201 (2004).

[6] A. Altmeyer, S. Riedl, C. Kohstall, M.J. Wright, R. Geursen, M. Bartenstein, C. Chin, J.H. Denschlag, and R. Grimm, Phys. Rev. Lett. 98, 040401 (2007); D.E. Miller, J.K. Chin, C.A. Stan, Y. Liu, W. Setiawan, C. Sanner, and W. Ketterle, Phys. Rev. Lett. 99, 070402 (2007).

[7] S. Inoue, M.R. Andrews, J. Stenger, H.-J. Miesner, D.M. Stamper-Kurn, and W. Ketterle, Nature (London) 392, 151 (1998).
[8] D.M. Eagles, Phys. Rev. 186, 456 (1969).

[9] A.J. Leggett, Quantum Liquids (Oxford Univ. Press, Oxford, 2006).

[10] P. Nozières and S. Schmitt-Rink, J. Low Temp. Phys. 59, 195 (1985).

[11] A. Perali, P. Pieri, and G.C. Strinati, Phys. Rev. Lett. 93, 100404 (2004).

[12] S. Stringari, Europhys. Lett. 65, 749 (2004).

[13] H. Hu, A. Minguzzi, X.J. Liu, and M.P. Tosi, Phys. Rev. Lett. 93, 190403 (2004).

[14] N. Manini and L. Salasnich, Phys. Rev. A 71, 033625 (2005); G. Diana, N. Manini, and L. Salasnich, Phys. Rev. A 73, 065601 (2006).

[15] A. Spuntarelli, P. Pieri and G.C. Strinati, Phys. Rev. Lett. 99, 040401 (2007).

[16] B.D. Josephson, Phys. Lett. 1, 251 (1962).

[17] A. Smerzi, S. Fantoni, S. Giovanazzi, and S.R. Shenoy, Phys. Rev. Lett. 79, 4950 (1997).

[18] M. Albiez, R. Gati, J. Fölling, S. Hunsmann, M. Cristiani, and M.K. Oberthaler, Phys. Rev. Lett. 95, 010402 (2005); F.S. Cataliotti, S. Burger, C. Fort, P. Maddaloni, F. Minardi, A. Trombettoni, A. Smerzi, M. Inguscio, Science 293, 843 (2001).

[19] Gh.-S. Paraoanu, M. Rodriguez, and P. Tormä, Phys. Rev. A 66, 041603(R) (2002). 
[20] M. Wouters, J. Tempere, and J.T. Devreese, Phys. Rev. A 70, 013616 (2004).

[21] S.K. Adhikari, e-preprint arXiv:0802.2231

[22] A. Barone and G. Paternò, Physics and Applications of the Josephson Effect (Wiley, New York, 1982).

[23] V.L. Ginzburg and L.D. Landau, Zh. Eksp. Teor. Fiz. 20, 1064 (1950).

[24] D.V. Averin, Nature 398, 748 (1999).

[25] A.M. Zagoskin, S. Ashhab, J.R. Johansson, F. Nori, Phys. Rev. Lett. 97, 077001 (2006).

[26] L. Salasnich, N. Manini, and A. Parola, Phys. Rev. A 72, 023621 (2005); L. Salasnich, Phys. Rev. A 76, 015601 (2007).

[27] L.D. Landau and E.M. Lifshitz, Statistical Physics: Theory of the Condensed State, (Pergamon, London, 1987).

[28] L.D. Landau and E.M. Lifshitz, Fluid mechanics (Pergamon, London, 1987).

[29] S. Giorgini, L.P. Pitaevskii, and S. Stringari, arXiv:0706.3360

[30] G.E. Astrakharchik, J. Boronat, J. Casulleras, and S. Giorgini, Phys. Rev. Lett. 93, 200404 (2004).
[31] I.J.R. Aitchison, P. Ao, D.J. Thouless, and X.-M. Zhu, Phys. Rev. B 51, 6531 (1995); S. De Palo, C. Castellani, C. Di Castro, and B. K. Chakraverty, Phys. Rev. B 60, 564 (1999).

[32] Notice that Spuntarelli, Pieri and Strinati [15] do not work in the regime of weak-link: in their calculations the energy barrier is much smaller than the Fermi energy.

[33] R.P. Feynman, R. Leighton, and M. Sands, Feynman Lectures On Physics, vol. 3 (Addison Wesley, Reading, 1966); R.P. Feynman, Statistical Mechanics. A set of Lectures (Benjamin, Reading, 1973).

[34] A. O. Caldeira and A. J. Leggett, Ann. Phys. (N.Y.) 149, 374 (1983).

[35] F. Meier and W. Zwerger, Phys. Rev. A 64, 033610 (2001).

[36] V. Ambegaokar and A. Baratoff, Phys. Rev. Lett. 10, 486 (1963); 11, 104 (1963).

[37] S. Backhaus, S. Pereverzev, R.W. Simmonds, A. Loshak, J.C. Davis, and R.E. Packard, Nature (London) 392, $687(1998)$. 